\title{
On higher-order adjacent derivative of perturbation map in parametric vector optimization
}

\section{Le Thanh Tung*}

"Correspondence:
Ittung@ctu.edu.vn
Department of Mathematics,
College of Natural Sciences, Can
Tho University, Cantho, Vietnam

\begin{abstract}
This paper deals with higher-order sensitivity analysis in terms of the higher-order adjacent derivative for nonsmooth vector optimization. The relations between the higher-order adjacent derivative of the minima/the proper minima/the weak minima of a multifunction and its profile map are given. Then the relationships between the higher-order adjacent derivative of the perturbation map/the proper perturbation map/the weak perturbation map, and the higher-order adjacent derivative of a feasible map in objective space are considered. Finally, the formulas for estimating the higher-order adjacent derivative of the perturbation map, the proper perturbation map, the weak perturbation map via the adjacent derivative of the constraint map, and the higher-order Fréchet derivative of the objective map are also obtained.
\end{abstract}

MSC: $90 \mathrm{C} 46 ; 49 J 52 ; 46 \mathrm{G} 05 ;$ 90C26; 90C29

Keywords: higher-order adjacent derivative; parameterized vector optimization problem; perturbation map; proper perturbation map; weak perturbation map; higher-order sensitivity analysis

\section{Introduction}

Sensitivity analysis provides quantitative information as regards the solution map of a parameterized multiobjective optimization problem. A number of interesting results have been obtained in sensitivity analysis for multiobjective optimization problems. One of the first results was given by Tanino in $[1,2]$. By using the first-order contingent derivative, some results concerning the behavior of perturbation maps were obtained. The TPderivative was presented in [3] and used to weaken some assumptions in [1, 4]. References [5-8] investigated the perturbation map in nonsmooth convex problems. In [9-11], the Clarke derivatives were used for analyzing the sensitivity. The concept of the protodifferentiability of a multifunction, in which the contingent cone coincides with the adjacent cone at a point to its graph, was presented by Rockafellar in [12]. In [13, 14], the important results on the proto-differentiability of the efficient solution maps were obtained for generalized equations, a general model including optimization problems. Some developments were obtained in $[15,16]$. A second-order sensitivity analysis via the secondorder contingent derivatives were considered in $[17,18]$. In [19], the second-order protodifferentiability of a multifunction was proposed to discuss the second-order sensitivity

(c) 2016 Tung. This article is distributed under the terms of the Creative Commons Attribution 4.0 International License (http://creativecommons.org/licenses/by/4.0/), which permits unrestricted use, distribution, and reproduction in any medium, provided you give appropriate credit to the original author(s) and the source, provide a link to the Creative Commons license, and indicate if changes were made. 
properties for generalized perturbation maps. The second-order radial-asymptotic derivative, introduced in [20], was used in qualification conditions to consider the second-order proto-differentiability of the efficient solution map and the efficient frontier map of a parameterized vector optimization problem in [21]. Some results in higher-order sensitivity analysis using a higher-order adjacent derivative in [22] and a higher-order contingent derivative in [23] of perturbation maps in a parameterized vector optimization were given. Using higher-order variational sets, presented in [24], some results in higher-order sensitivity analysis were obtained in [25].

Unlike higher-order contingent derivatives based on encounter information, the $m$ thorder variations of a map, based on the different rates of change of the point under consideration in the domain space and the range space of a map, were proposed to obtain the open mapping principle in [26] and consider Hölder metric regularity of set-valued maps in [27]. Another kind of $m$ th-order derivatives, presented in [28], was used to establish the optimality condition for isolated local minima of nonsmooth functions and modified to characterize weak sharp minima in [29]. The $m$ th-order derivatives in [28] were generalized to set-valued maps in [30-32] to establish higher-order optimality conditions. In [31], the higher-order sensitivity was consider by using the $m$ th-order contingent-type derivatives. In [33], the lower Studniarski derivative of a perturbation map in vector optimization was considered.

To the best of our knowledge, there is no paper dealing with the sensitivity of the $m$ thorder adjacent derivatives of perturbation maps of parameterized vector optimization problems. Moreover, the proper perturbation maps and the case that the objective function is higher-order Fréchet differentiable in constraint vector optimization have not been considered yet. Motivated by the above observations, in this paper, by making use of the $m$ th-order adjacent derivatives of set-valued maps which were introduced in [31], we investigate quantitatively the perturbation map, the proper perturbation map, and the weak perturbation map of parameterized vector optimization problems. The paper is organized as follows. Section 2 contains preliminary facts we need in the paper. In Section 3, the relations between the $m$ th-order adjacent derivatives of a set-valued map and those of its profile map are discussed. The obtained results are employed in Section 4 to investigate the relationships between the $m$ th-order adjacent derivatives of the perturbation map/the proper perturbation map/the weak perturbation map and the $m$ th-order adjacent derivative of the feasible map in the objective space. In Section 4, the formulas for estimating the $m$ th-order adjacent derivatives of the perturbation map, the proper perturbation map, and the weak perturbation map via the adjacent derivative of constraint map and the $m$ thorder Fréchet derivative of the objective map are also given.

\section{Preliminaries}

In this paper, if not otherwise stated, let $X, Y$, and $Z$ be normed spaces, and $C \subseteq Y$ be a pointed closed convex cone. $\mathcal{U}\left(x_{0}\right)$ is used for the set of neighborhoods of $x_{0} . \mathbb{R}, \mathbb{R}_{+}$, and $\mathbb{N}$ stand for the set of the real numbers, nonnegative real numbers, and natural numbers, respectively (shortly, resp.). For $M \subseteq X$, int $M, \operatorname{cl} M$, bd $M$ denote its interior, closure, and boundary, resp. A convex set $B \subseteq Y$ is called a base of $C$ iff $0 \notin \operatorname{cl} B$ and $C=\{t b \mid t \in$ $\left.\mathbb{R}_{+}, b \in B\right\}$. Clearly $C$ has a compact base $B$ if and only if $C \cap \operatorname{bd} B$ is compact. If $Y$ is a finite dimensional space, then $C$ has a compact base. For $F: X \rightrightarrows Y$, the domain, graph, 
and epigraph of $F$ are defined by, resp.,

$$
\begin{aligned}
& \operatorname{dom} F:=\{x \in X \mid F(x) \neq \emptyset\}, \quad \operatorname{gr} F:=\{(x, y) \in X \times Y \mid y \in F(x)\}, \\
& \operatorname{epi} F:=\{(x, y) \in X \times Y \mid y \in F(x)+C\} .
\end{aligned}
$$

The profile map of $F$ is $F+C$ (defined by $(F+C)(x):=F(x)+C$ ). We recall some concepts of optimality/efficiency in vector optimization as follows, for $a_{0} \in A \subseteq Y$.

(i) $a_{0}$ is called a local (Pareto) minimal/efficient point of $A$ (with respect to $C$ ), and denoted by $a_{0} \in \operatorname{Min}_{C} A$, iff there exists $U \in \mathcal{U}\left(a_{0}\right)$ such that

$$
\left(A \cap U-a_{0}\right) \cap(-C \backslash\{0\})=\emptyset .
$$

(ii) Supposing that int $C \neq \emptyset, a_{0}$ is said to be a local weak minimal/efficient point of $A$, denoted by $a \in \operatorname{WMin}_{C} A$, iff there exists $U \in \mathcal{U}\left(a_{0}\right)$ such that

$$
\left(A \cap U-a_{0}\right) \cap(-\operatorname{int} C)=\emptyset .
$$

(iii) Assuming that $C$ is pointed, $a_{0}$ is termed a proper minimal/efficient point of $A$, denoted by $a_{0} \in \operatorname{PrMin}_{C} A$, iff there exists a convex cone $K \varsubsetneqq Y$ with $C \backslash\{0\} \subseteq \operatorname{int} K$ and $U \in \mathcal{U}\left(a_{0}\right)$ such that

$$
\left(A \cap U-a_{0}\right) \cap(-K)=\{0\}
$$

If $U=Y$, the word 'local' is omitted, i.e., we have the corresponding global notions. For a subset $A \subseteq Y, A$ is said to have the domination property iff $A \subseteq \operatorname{Min}_{C} A+C$ and $A$ is said to have the proper domination property iff $A \subseteq \operatorname{PrMin}_{C} A+C$. Similarly, when int $C \neq \emptyset$, $A$ has the weak domination property iff $A \subseteq \mathrm{WMin}_{C} A+\operatorname{int} C \cup\{0\}$.

Recall now the four kinds of higher-order derivatives which we are most concerned with in the sequel. Let $F: X \rightrightarrows Y, u \in X, m \in \mathbb{N}$, and $\left(x_{0}, y_{0}\right) \in \operatorname{gr} F$.

(i) ([31]) The $m$ th-order radial-contingent derivative of $F$ at $\left(x_{0}, y_{0}\right)$ is defined by

$$
\begin{aligned}
D_{S}^{m} F\left(x_{0}, y_{0}\right)(u):= & \left\{v \in Y \mid \exists t_{n}>0, \exists\left(u_{n}, v_{n}\right) \rightarrow(u, v): t_{n} u_{n} \rightarrow 0,\right. \\
& \left.y_{0}+t_{n}^{m} v_{n} \in F\left(x_{0}+t_{n} u_{n}\right)\right\} .
\end{aligned}
$$

(ii) ([32]) The $m$ th-order contingent-type derivative of $F$ at $\left(x_{0}, y_{0}\right)$ is defined by

$$
D^{m} F\left(x_{0}, y_{0}\right)(u):=\left\{v \in Y \mid \exists t_{n} \downarrow 0, \exists\left(u_{n}, v_{n}\right) \rightarrow(u, v), y_{0}+t_{n}^{m} v_{n} \in F\left(x_{0}+t_{n} u_{n}\right)\right\} .
$$

(iii) ([31]) The $m$ th-order adjacent derivative of $F$ at $\left(x_{0}, y_{0}\right)$ is defined by

$$
D^{b m} F\left(x_{0}, y_{0}\right)(u):=\left\{v \in Y \mid \forall t_{n} \downarrow 0, \exists\left(u_{n}, v_{n}\right) \rightarrow(u, v), y_{0}+t_{n}^{m} v_{n} \in F\left(x_{0}+t_{n} u_{n}\right)\right\} .
$$

(iv) ([32]) The $m$ th-order lower Studniarski derivative of $F$ at $\left(x_{0}, y_{0}\right)$ is defined by

$$
D^{l m} F\left(x_{0}, y_{0}\right)(u):=\left\{v \in Y \mid \forall t_{n} \downarrow 0, \forall u_{n} \rightarrow u, \exists v_{n} \rightarrow v, y_{0}+t_{n}^{m} v_{n} \in F\left(x_{0}+t_{n} u_{n}\right)\right\} .
$$


$\operatorname{Remark} 2.1 D^{l m} F\left(x_{0}, y_{0}\right)(u) \subseteq D^{b m} F\left(x_{0}, y_{0}\right)(u) \subseteq D^{m} F\left(x_{0}, y_{0}\right)(u), \forall u \in X$.

The reverse conclusions in Remark 2.1 may not hold. The following examples show the cases.

Example 2.1 Let $\mathcal{I}=\left\{\frac{1}{n}: n \in \mathbb{N}\right\}$ and $F: \mathbb{R} \rightrightarrows \mathbb{R}$ be defined by

$$
F(x)= \begin{cases}\{0\}, & \text { if } x \leq 0 \\ \left\{-x^{2}\right\}, & \text { if } x \in \mathcal{I} \\ \emptyset, & \text { otherwise }\end{cases}
$$

Then, for $\left(x_{0}, y_{0}\right)=(0,0) \in \operatorname{gr} F$, we can check that

$$
D^{2} F\left(x_{0}, y_{0}\right)(2)=\{-4\}
$$

Taking $t_{n}=\frac{2}{n}$, then $t_{n} u_{n}=\frac{2 u_{n}}{n} \nsubseteq \mathcal{I}$ for all $u_{n} \rightarrow 2$. Indeed, suppose to the contrary that there exists a subsequence $\left\{\frac{1}{k}\right\} \subseteq \mathcal{I}, k \geq n$, such that $t_{n} u_{n}=\frac{1}{k}$. Then $u_{n}=\frac{1}{2} \cdot \frac{n}{k} \leq \frac{1}{2}$, i.e., $u_{n} \nrightarrow 2$, a contradiction. Hence, for the above $t_{n}, F\left(x_{0}+t_{n} u_{n}\right)=\emptyset$. Consequently,

$$
D^{b 2} F\left(x_{0}, y_{0}\right)(2)=\emptyset
$$

Hence,

$$
D^{2} F\left(x_{0}, y_{0}\right)(2) \nsubseteq D^{b 2} F\left(x_{0}, y_{0}\right)(2) \text {. }
$$

Example 2.2 Let $F: \mathbb{R} \rightrightarrows \mathbb{R}^{2}$ be defined by

$$
F(x)= \begin{cases}\left\{\left(y_{1}, y_{2}\right) \in \mathbb{R}^{2}: y_{1} \leq x^{4}, y_{2} \leq 0\right\}, & \text { if } x<0 \\ \left\{\left(y_{1}, y_{2}\right) \in \mathbb{R}^{2}: y_{1} \leq 0, y_{2} \geq 2 x^{4}\right\}, & \text { if } x \geq 0\end{cases}
$$

Then, for $\left(x_{0}, y_{0}\right)=(0,(0,0))$,

$$
D^{b 2} F\left(x_{0}, y_{0}\right)(x)= \begin{cases}\left\{\left(y_{1}, y_{2}\right) \in \mathbb{R}^{2}: y_{1} \leq 0, y_{2} \leq 0\right\}, & \text { if } x<0, \\ \left\{\left(y_{1}, y_{2}\right) \in \mathbb{R}^{2}: y_{1} \leq 0, y_{2} \in \mathbb{R}\right\}, & \text { if } x=0, \\ \left\{\left(y_{1}, y_{2}\right) \in \mathbb{R}^{2}: y_{1} \leq 0, y_{2} \geq 0\right\}, & \text { if } x>0,\end{cases}
$$

and

$$
D^{l 2} F\left(x_{0}, y_{0}\right)(x)= \begin{cases}\left\{\left(y_{1}, y_{2}\right) \in \mathbb{R}^{2}: y_{1} \leq 0, y_{2} \leq 0\right\}, & \text { if } x<0, \\ \left\{\left(y_{1}, y_{2}\right) \in \mathbb{R}^{2}: y_{1} \leq 0, y_{2}=0\right\}, & \text { if } x=0, \\ \left\{\left(y_{1}, y_{2}\right) \in \mathbb{R}^{2}: y_{1} \leq 0, y_{2} \geq 0\right\}, & \text { if } x>0\end{cases}
$$

Hence,

$$
D^{b 2} F\left(x_{0}, y_{0}\right)(0) \nsubseteq D^{l 2} F(\bar{x}, \bar{y})(0) .
$$

Definition 2.1 (see [31]) For $u \in X, F: X \rightrightarrows Y$ is called $m$ th-order $u$-directionally contingent compact at $\left(x_{0}, y_{0}\right) \in \operatorname{gr} F$ iff, for any $t_{n} \downarrow 0,\left(u_{n}, v_{n}\right) \in X \times Y$ such that $u_{n} \rightarrow u$, and $y_{0}+t_{n}^{m} v_{n} \in F\left(x_{0}+t_{n} u_{n}\right)$ for all $n$, there exists a convergent subsequence of $\left\{v_{n}\right\}$. 
Definition 2.2 Let $F: X \rightrightarrows Y$ be a set-valued map, $x_{0} \in X, m \in \mathbb{N} \backslash\{0\}$, and $\alpha>0$.

(i) $F$ is said locally Hölder continuous of order $\alpha$ at $\left(x_{0}, y_{0}\right) \in \operatorname{gr} F$ if there exist $\lambda>0$, and $U \in \mathcal{U}\left(x_{0}\right)$ such that

$$
F\left(x_{2}\right) \subset F\left(x_{1}\right)+\lambda\left\|x_{1}-x_{2}\right\|^{\alpha} B_{Y}, \quad \forall x_{1}, x_{2} \in U,
$$

where $B_{Y}$ stands for the closed unit ball in $Y$.

(ii) $F$ is said locally pseudo-Hölder calm of order $m$ (see [34]) at $\left(x_{0}, y_{0}\right) \in \operatorname{gr} F$ if there exist a real number $\lambda>0, \exists U \in \mathcal{U}\left(x_{0}\right)$, and $\exists V \in \mathcal{U}\left(y_{0}\right)$ such that

$$
F(x) \cap V \subset\left\{y_{0}\right\}+\lambda\left\|x-x_{0}\right\|^{m} B_{Y}, \quad \forall x \in U .
$$

When $m=1$, the word 'Hölder' is replaced by 'Lipschitz'. If $V=Y$, then 'locally pseudoHölder calm' is replaced by 'locally Hölder calm'. In [13], $F$ is called upper locally Lipschitz at $x_{0} \in \operatorname{dom} F$ if there exist a real number $\lambda>0$ and $U \in \mathcal{U}\left(x_{0}\right)$ such that $F(x) \subset F\left(x_{0}\right)+\lambda \| x-$ $x_{0} \| B_{Y}, \forall x \in U$. It is easy to see that if $F$ is upper locally Lipschitz at $x_{0}$ and $F\left(x_{0}\right)=\left\{y_{0}\right\}$ then $F$ is locally Lipschitz calm at $\left(x_{0}, y_{0}\right)$.

Proposition 2.1 (see [31]) Let $F: X \rightrightarrows Y,\left(x_{0}, y_{0}\right) \in \operatorname{gr} F$, and $Y$ be finite dimensional space. If $D_{S}^{m} F\left(x_{0}, y_{0}\right)(0)=\{0\}$, then $F$ is mth-order $u$-directionally contingent compact at $\left(x_{0}, y_{0}\right)$ for all $u \in X$.

Proposition 2.2 Let $F: X \rightrightarrows Y,\left(x_{0}, y_{0}\right) \in \operatorname{gr} F$, and $Y$ be finite dimensional space. If $F$ is locally Hölder calm of order $m$ at $\left(x_{0}, y_{0}\right) \in \operatorname{gr} F$, then $D_{S}^{m} F\left(x_{0}, y_{0}\right)(0)=\{0\}$.

Proof Consider an arbitrary $y \in D_{S}^{m} F\left(x_{0}, y_{0}\right)(0)$. Then there exist $y_{n} \rightarrow y, x_{n} \rightarrow 0$, and $t_{n}>$ 0 such that $y_{0}+t_{n}^{m} y_{n} \in F\left(x_{0}+t_{n} x_{n}\right)$ and $t_{n} x_{n} \rightarrow 0$. Since $F$ is locally Hölder calm of order $m$ at $\left(x_{0}, y_{0}\right)$, we derive that, for $n$ large enough, there exists $\lambda>0$ such that

$$
y_{0}+t_{n}^{m} y_{n} \in y_{0}+\lambda\left\|t_{n} x_{n}\right\|^{m} B_{Y}
$$

Consequently,

$$
y_{n} \in \lambda\left\|x_{n}\right\|^{m} B_{Y} .
$$

It follows from the above equation that $x_{n} \rightarrow 0$, and $y_{n} \rightarrow y$ that one has $y=0$.

Corollary 2.1 Let $F: X \rightrightarrows Y,\left(x_{0}, y_{0}\right) \in \operatorname{gr} F$, and $Y$ be finite dimensional space. If $F$ is locally Hölder calm of order $m$ at $\left(x_{0}, y_{0}\right) \in \operatorname{gr} F$, then $F$ is mth-order u-directionally contingent compact at $\left(x_{0}, y_{0}\right)$ for all $u \in X$.

Proof It follows from Proposition 2.1 and Proposition 2.2 that the conclusion is obtained.

Definition 2.3 (see [31]) Let $f: X \rightarrow Y$ be a vector-valued map. $f$ is said to be $m$ th-order Fréchet differentiable at $x_{0} \in X$ iff there exists a linear continuous operator $d^{m} F\left(x_{0}\right): X \times$ 
$\cdots \times X \rightarrow Y(m$ times $X)$, such that

$$
f(x)=f\left(x_{0}\right)+d^{m} f\left(x_{0}\right)\left(x-x_{0}, \ldots, x-x_{0}\right)+o\left(\left\|x-x_{0}\right\|^{m}\right) \quad\left(m \text { times } x-x_{0}\right),
$$

where $o\left(\left\|x-x_{0}\right\|^{m}\right)$ satisfies $o\left(\left\|x-x_{0}\right\|^{m}\right) /\left\|x-x_{0}\right\|^{m} \rightarrow 0$ when $x \rightarrow x_{0} . d^{m} f\left(x_{0}\right)$ is called the $m$ th-order Fréchet derivative. $f$ is said $m$ th-order Fréchet differentiable on $X$ if $f$ is $m$ th-order Fréchet differentiable at any $x \in X$. If $d^{m} f(\cdot)$ is continuous at $x_{0}$ then $f$ is said to be $m$ th-order continuously Fréchet differentiable at $x_{0}$.

Remark 2.2 (see [31]) For $f: X \rightarrow Y$ and $x_{0}, u \in X$, if there exists $d^{m} f\left(x_{0}\right)$, then

$$
\begin{aligned}
\left\{d^{m} f\left(x_{0}\right)(u, u, \ldots, u)\right\} & =D^{l m} f\left(x_{0}, f\left(x_{0}\right)\right)(u)=D^{b m} f\left(x_{0}, f\left(x_{0}\right)\right)(u) \\
& =D^{m} f\left(x_{0}, f\left(x_{0}\right)\right)(u)=D_{S}^{m} f\left(x_{0}, f\left(x_{0}\right)\right)(u) .
\end{aligned}
$$

\section{Higher-order adjacent derivatives of set-valued maps}

In this section, the relations between higher-order adjacent derivative of a set-valued map and those of its profile map are discussed. Such relations for various kinds of efficient points of these derivatives are also investigated.

Proposition 3.1 Let $\left(x_{0}, y_{0}\right) \in \operatorname{gr} F$. Then, for any $u \in X$,

$$
D^{b m} F\left(x_{0}, y_{0}\right)(u)+C \subseteq D^{b m}(F+C)\left(x_{0}, y_{0}\right)(u)
$$

Proof Let $z=v+c$ for some $v \in D^{b m} F\left(x_{0}, y_{0}\right)(u)$ and $c \in C$. Then, for all $t_{n} \downarrow 0$, there exists $\left(u_{n}, v_{n}\right) \rightarrow(u, v)$ such that $y_{0}+t_{n}^{m} v_{n} \in F\left(x_{0}+t_{n} u_{n}\right)$ for all $n$. Setting $\bar{v}_{n}:=v_{n}+c$, one has $\bar{v}_{n} \rightarrow v+c$ and, for all $n$,

$$
y_{0}+t_{n}^{m} \bar{v}_{n}=y_{0}+t_{n}^{m}\left(v_{n}+c\right) \in F\left(x_{0}+t_{n} u_{n}\right)+C=(F+C)\left(x_{0}+t_{n} u_{n}\right) .
$$

So, $z=v+c \in D^{b m}(F+C)\left(x_{0}, y_{0}\right)(u)$.

Note that the opposite inclusion of (1) may not hold. The following example illustrates the case.

Example 3.1 Let $C=\mathbb{R}_{+}, F: \mathbb{R} \rightrightarrows \mathbb{R}$ be defined by

$$
F(x)= \begin{cases}\left\{-1, x^{3}\right\}, & \text { if } x \geq 0 \\ \{0\}, & \text { if } x<0\end{cases}
$$

Let $\left(x_{0}, y_{0}\right)=(0,0) \in \operatorname{gr} F$. Then

$$
D^{b 3} F(0,0)(u)=\left\{\begin{array}{ll}
\left\{u^{3}\right\}, & \text { if } u \geq 0, \\
\{0\}, & \text { if } u<0,
\end{array} \quad D^{b 3}(F+C)(0,0)(u)= \begin{cases}\mathbb{R}, & \text { if } u \geq 0, \\
\mathbb{R}_{+}, & \text {if } u<0 .\end{cases}\right.
$$

Hence, for all $u$,

$$
D^{b 3}(F+C)\left(x_{0}, y_{0}\right)(u) \nsubseteq D^{b 3} F\left(x_{0}, y_{0}\right)(u)+C .
$$


Proposition 3.2 Suppose that either of the following conditions is satisfied:

(i) for any $u \in X, F$ is mth-order $u$-directionally contingent compact at $\left(x_{0}, y_{0}\right)$;

(ii) $C$ has a compact base and $D_{S}^{m} F\left(x_{0}, y_{0}\right)(0) \cap(-C)=\{0\}$;

(iii) $C$ has a compact base and $D^{b m}(F+C)\left(x_{0}, y_{0}\right)(u)$ has domination property.

Then, for all $u \in X$,

$$
D^{b m} F\left(x_{0}, y_{0}\right)(u)+C=D^{b m}(F+C)\left(x_{0}, y_{0}\right)(u) .
$$

Proof It follows from Proposition 3.1 that we only need to show the reverse inclusion of (1).

(i) Let $v \in D^{b m}(F+C)\left(x_{0}, y_{0}\right)(u)$. If $(u, v)=(0,0)$, we have $0 \in D^{b m} F\left(x_{0}, y_{0}\right)(0)+C$. For $(u, v) \neq(0,0)$, for all $t_{n} \downarrow 0$, there exists $\left(u_{n}, v_{n}\right) \rightarrow(u, v)$ such that $y_{0}+t_{n}^{m} v_{n} \in(F+C)\left(x_{0}+\right.$ $\left.t_{n} u_{n}\right), \forall n$. Hence, there exists $c_{n} \in C$ such that $y_{0}+t_{n}^{m}\left(v_{n}-c_{n} / t_{n}^{m}\right) \in F\left(x_{0}+t_{n} u_{n}\right)$. By the $u$-directionally contingent compactness of $F$ at $\left(x_{0}, y_{0}\right)$, we can assume that $v_{n}-c_{n} / t_{n}^{m} \rightarrow$ $\bar{v} \in D^{b m} F\left(x_{0}, y_{0}\right)(u)$. Since $c_{n} / t_{n}^{m}=v_{n}-\left(v_{n}-c_{n} / t_{n}^{m}\right) \rightarrow v-\bar{v}$ and $C$ is a closed convex cone, one gets $v-\bar{v} \in C$. Hence, $v \in \bar{v}+C \subseteq D^{b m} F\left(x_{0}, y_{0}\right)(u)+C$.

(ii) Let $u \in X$ and $v \in D^{b m}(F+C)\left(x_{0}, y_{0}\right)(u)$ be arbitrary. As in (i), we need to consider only $(u, v) \neq(0,0)$. We see that, for all $t_{n} \downarrow 0$, there exist $\left(u_{n}, v_{n}\right) \rightarrow(u, v)$, and $c_{n} \in C$ such that $y_{0}+t_{n}^{m} v_{n} \in F\left(x_{0}+t_{n} u_{n}\right)+c_{n}$ for all $n$. If there exists $n_{0}$ such that $c_{n}=0$ for all $n>n_{0}$, then $v \in D^{b m} F\left(x_{0}, y_{0}\right)(u)+0 \subseteq D^{b m} F\left(x_{0}, y_{0}\right)(u)+C$. Now, assume that $c_{n} \neq 0$ and $c_{n} /\left\|c_{n}\right\| \rightarrow \bar{c}$ for some $\bar{c} \in C$ with norm one. There are only two cases for $s_{n}:=\sqrt[m]{\left\|c_{n}\right\|}>0$.

Case 1: $s_{n} / t_{n} \rightarrow+\infty$. Then $s_{n}\left[\left(t_{n} / s_{n}\right) u_{n}\right]=t_{n} u_{n} \rightarrow 0$. Since

$$
y_{0}+\left(s_{n}\right)^{m}\left[\left(t_{n} / s_{n}\right)^{m} v_{n}-c_{n} / s_{n}^{m}\right] \in F\left(x_{0}+s_{n}\left[\left(t_{n} / s_{n}\right) u_{n}\right]\right)
$$

$\left(t_{n} / s_{n}\right)^{m} y_{n}-c_{n} / s_{n}^{m} \rightarrow-\bar{c}$, and $\left(t_{n} / s_{n}\right) u_{n} \rightarrow 0$, one has $-\bar{c} \in D_{S}^{m} F\left(x_{0}, y_{0}\right)(0)$, an impossibility.

Case 2: $\left\{s_{n} / t_{n}\right\}$ is bounded, and assume $s_{n} / t_{n} \rightarrow \alpha \geq 0$. Then, since

$$
y_{0}+\left(t_{n}\right)^{m}\left[v_{n}-\left(s_{n} / t_{n}\right)^{m}\left(c_{n} / s_{n}^{m}\right)\right] \in F\left(x_{0}+t_{n} u_{n}\right),
$$

$v_{n}-\left(s_{n} / t_{n}\right)^{m}\left(c_{n} / s_{n}^{m}\right) \rightarrow v-\alpha^{m} \bar{c}$, and $u_{n} \rightarrow u$, one gets $v-\alpha^{m} \bar{c} \in D^{b m} F\left(x_{0}, y_{0}\right)(u)$, and hence $v \in D^{b m} F\left(x_{0}, y_{0}\right)(u)+C$.

(iii) Since $D^{b m}(F+C)\left(x_{0}, y_{0}\right)(u)$ has the domination property, for any $u \in X$,

$$
D^{b m}(F+C)\left(x_{0}, y_{0}\right)(u) \subseteq \operatorname{Min}_{C} D^{b m}(F+C)\left(x_{0}, y_{0}\right)(u)+C .
$$

We claim that, for any $u \in X$,

$$
\operatorname{Min}_{C} D^{b m}(F+C)\left(x_{0}, y_{0}\right)(u) \subseteq D^{b m} F\left(x_{0}, y_{0}\right)(u) .
$$

Indeed, let $v \in \operatorname{Min}_{C} D^{b m}(F+C)\left(x_{0}, y_{0}\right)(u)$. Then, for all $t_{n} \downarrow 0$, there exist $\left(u_{n}, v_{n}\right) \rightarrow(u, v)$ and $c_{n} \in C$ such that, for all $n, y_{0}+t_{n}^{m}\left(v_{n}-c_{n}\right) \in F\left(x_{0}+t_{n} u_{n}\right)$. Since $C$ has a compact base, we may assume that $c_{n}=\alpha_{n} b_{n}$ with $\alpha_{n}>0$ and $b_{n} \rightarrow b \neq 0$. We now show that $\alpha_{n} \rightarrow 0$. Suppose to the contrary that $\alpha_{n} \not>0$. Then there exists $\epsilon>0$ such that $\alpha_{n} \geq \epsilon$ for all $n$. Setting $\bar{c}_{n}=\left(\epsilon / \alpha_{n}\right) c_{n}$. Then, for any $n, c_{n}-\bar{c}_{n}=\left(1-\epsilon / \alpha_{n}\right) c_{n} \in C$ and

$$
y_{0}+t_{n}^{m}\left(v_{n}-\bar{c}_{n}\right)=y_{0}+t_{n}^{m}\left(v_{n}-c_{n}\right)+t_{n}^{m}\left(c_{n}-\bar{c}_{n}\right) \in F\left(x_{0}+t_{n} u_{n}\right)+C=(F+C)\left(x_{0}+t_{n} u_{n}\right) .
$$


Since $v_{n}-\bar{c}_{n}=v_{n}-\left(\epsilon / \alpha_{n}\right) c_{n}=v_{n}-\epsilon b_{n} \rightarrow v-\epsilon b$, we have $v-\epsilon b \in D^{b m}(F+C)\left(x_{0}, y_{0}\right)(u)$ and $v-(v-\epsilon b)=\epsilon b \in C \backslash\{0\}$, which contradicts $v \in \operatorname{Min}_{C} D^{b m}(F+C)\left(x_{0}, y_{0}\right)(u)$. Therefore, $\alpha_{n} \rightarrow 0$ and $v_{n}-c_{n}=v_{n}-\alpha_{n} b_{n} \rightarrow v$, i.e., $v \in D^{b m} F\left(x_{0}, y_{0}\right)(u)$. Thus, (3) holds. It follows from (2) and (3) that

$$
D^{b m}(F+C)\left(x_{0}, y_{0}\right)(u) \subseteq D^{b m} F\left(x_{0}, y_{0}\right)(u)+C, \quad \forall u \in X
$$

The proof is complete.

Proposition 3.3 Suppose that either of the following conditions holds:

(i) for any $u \in X, F$ is $m$ th-order $u$-directionally contingent compact at $\left(x_{0}, y_{0}\right)$;

(ii) $C$ has a compact base and $D_{S}^{m} F\left(x_{0}, y_{0}\right)(0) \cap(-C)=\{0\}$;

(iii) $C$ has a compact base and $D^{b m}(F+C)\left(x_{0}, y_{0}\right)(u)$ has domination property.

Then, for all $u \in X$,

$\operatorname{Min}_{C} D^{b m} F\left(x_{0}, y_{0}\right)(u)=\operatorname{Min}_{C} D^{b m}(F+C)\left(x_{0}, y_{0}\right)(u)$.

Proof Similarly to the proof of Proposition 4.3 in [31], we obtain the conclusion.

Since the following propositions are proven similarly, the proofs are omitted.

Proposition 3.4 Suppose that either of the following conditions holds:

(i) for any $u \in X, F$ is mth-order $u$-directionally contingent compact at $\left(x_{0}, y_{0}\right)$;

(ii) $C$ has a compact base and $D_{S}^{m} F\left(x_{0}, y_{0}\right)(0) \cap(-C)=\{0\}$;

(iii) $C$ has a compact base and $D^{b m}(F+C)\left(x_{0}, y_{0}\right)(u)$ has proper domination property.

Then, for all $u \in X$,

$\operatorname{PrMin}_{C} D^{b m} F\left(x_{0}, y_{0}\right)(u)=\operatorname{PrMin}_{C} D^{b m}(F+C)\left(x_{0}, y_{0}\right)(u)$.

Proposition 3.5 Assume that int $C \neq \emptyset$ and $\widetilde{K}$ is a closed convex cone with $\widetilde{K} \subseteq$ int $C \cup\{0\}$. Suppose further that either of the following conditions holds:

(i) for any $u \in X, F$ is mth-order $u$-directionally contingent compact at $\left(x_{0}, y_{0}\right)$;

(ii) $C$ has a compact base and $D_{S}^{m} F\left(x_{0}, y_{0}\right)(0) \cap(-\widetilde{K})=\{0\}$;

(iii) $C$ has a compact base and $D^{b m}(F+\widetilde{K})\left(x_{0}, y_{0}\right)(u)$ has weak domination property.

Then, for all $u \in X$,

$\operatorname{WMin}_{C} D^{b m} F\left(x_{0}, y_{0}\right)(u)=\operatorname{WMin}_{C} D^{b m}(F+\widetilde{K})\left(x_{0}, y_{0}\right)(u)$.

The following example illustrates that we cannot replace $\widetilde{K}$ by $C$ in the conclusion of Proposition 3.5 .

Example 3.2 Let $X=\mathbb{R}, Y=\mathbb{R}^{2},\left(x_{0}, y_{0}\right)=(0,(0,0)), C=\mathbb{R}_{+}^{2}$, and $F: \mathbb{R} \rightrightarrows \mathbb{R}^{2}$ be defined by $F(x)=\left\{\left(x^{2}, x^{4}\right)\right\}$. Then we can check that $D_{S}^{2} F\left(x_{0}, y_{0}\right)(u)=D^{2 b} F\left(x_{0}, y_{0}\right)(u)=\left\{\left(u^{2}, 0\right)\right\}$ for any $u \in \mathbb{R}$, and

$$
D^{2 b}(F+C)\left(x_{0}, y_{0}\right)(u)=\left\{\left(y_{1}, y_{2}\right) \in \mathbb{R}_{+}^{2}: y_{1} \geq u^{2}, y_{2} \geq 0\right\}
$$


Hence, $\operatorname{WMin}_{C} D^{2 b} F\left(x_{0}, y_{0}\right)(u)=\left\{\left(u^{2}, 0\right)\right\}$ and

$$
\begin{aligned}
& \operatorname{WMin}_{C} D^{2 b}(F+C)\left(x_{0}, y_{0}\right)(u) \\
& \quad=\left\{\left(y_{1}, y_{2}\right) \in \mathbb{R}_{+}^{2} \mid y_{1} \geq u^{2}, y_{2}=0\right\} \cup\left\{\left(y_{1}, y_{2}\right) \in \mathbb{R}_{+}^{2} \mid y_{1}=u^{2}, y_{2} \geq 0\right\} .
\end{aligned}
$$

Since $D_{S}^{2} F\left(x_{0}, y_{0}\right)(0) \cap(-C)=\{0\}$ and $C$ has a compact base $B=\left\{\left(y_{1}, y_{2}\right) \in \mathbb{R}^{2}, y_{1}+y_{2}=\right.$ $\left.1, y_{1} \geq 0, y_{2} \geq 0\right\}$, the assumption (ii) is fulfilled. We can check that

$$
\mathrm{WMin}_{C} D^{2 b}(F+C)\left(x_{0}, y_{0}\right)(u) \nsubseteq \mathrm{WMin}_{C} D^{2 b} F\left(x_{0}, y_{0}\right)(u) .
$$

\section{Higher-order adjacent derivatives of perturbations maps}

In this section, we consider the following parameterized vector optimization problem:

$$
\operatorname{Min}_{K} f(x, u)=\left(f_{1}(x, u), f_{2}(x, u), \ldots, f_{q}(x, u)\right), \quad \text { s.t. } x \in X(u) \subseteq \mathbb{R}^{l},
$$

where $x$ is a $l$-dimensional decision variable, $u$ is a $p$-dimensional parameter, $f_{i}$ is a real valued objective function on $\mathbb{R}^{l} \times \mathbb{R}^{p}$ for $i=1,2, \ldots, q, X$ is a set-valued map from $\mathbb{R}^{p}$ to $\mathbb{R}^{l}$, which defines a feasible decision set, and $K$ is a nonempty pointed closed convex ordering cone in $\mathbb{R}^{q}$. Let $F(u)$ be the value at $u$ of the feasible set map in the objective space, i.e.,

$$
F(u):=\left\{y \in \mathbb{R}^{q} \mid y=f(x, u) \text { for some } x \in X(u)\right\} .
$$

We define the perturbation/frontier map $\mathcal{F}$, the weak perturbation/frontier map $\mathcal{W}$, and the proper perturbation/frontier map $\mathcal{P}$ of the considered problem as follows:

$$
\mathcal{F}(u):=\operatorname{Min}_{K} F(u), \quad \mathcal{W}(u):=\operatorname{WMin}_{K} F(u), \quad \mathcal{P}(u):=\operatorname{PrMin}_{K} F(u) .
$$

For $u_{0} \in \mathbb{R}^{p}$ and a closed convex cone $\widetilde{K} \subseteq \mathbb{R}^{q}$,

(i) $F$ is said to be $K$-dominated by $\mathcal{F}$ near $u_{0}$ iff $F(u) \subseteq \mathcal{F}(u)+K$, for all $u$ in some $U \in \mathcal{U}\left(u_{0}\right)$.

(ii) $F$ is said to be $K$-dominated by $\mathcal{P}$ near $u_{0}$ iff $F(u) \subseteq \mathcal{P}(u)+K$, for all $u$ in some $U \in \mathcal{U}\left(u_{0}\right)$.

(iii) $F$ is said to be $\widetilde{K}$-dominated by $\mathcal{W}$ near $u_{0}$ iff $F(u) \subseteq \mathcal{W}(u)+\widetilde{K}$, for all $u$ in some $U \in \mathcal{U}\left(u_{0}\right)$.

Remark 4.1 Since $\mathcal{F}(u) \subseteq F(u)$, the $K$-dominatedness of $F$ by $\mathcal{F}$ near $u_{0}$ implies that, for all $u \in U, \mathcal{F}(u)+K=F(u)+K$. Hence, if $F$ is $K$-dominated by $\mathcal{F}$ near $u_{0}$, then for any $u_{0} \in \mathbb{R}^{p}, y_{0} \in \mathcal{F}\left(u_{0}\right)$, and $u \in U$,

$$
D^{b m}(\mathcal{F}+K)\left(u_{0}, y_{0}\right)(u)=D^{b m}(F+K)\left(u_{0}, y_{0}\right)(u) .
$$

Similar assertions are true for $\mathcal{P}$ and $\mathcal{W}$ as follows.

(i) $D^{b m}(\mathcal{P}+K)\left(u_{0}, y_{0}\right)(u)=D^{b m}(F+K)\left(u_{0}, y_{0}\right)(u)$ for any $u_{0} \in \mathbb{R}^{p}, y_{0} \in \mathcal{P}\left(u_{0}\right)$, and $u \in U$ if $F$ is $K$-dominated by $\mathcal{P}$ near $u_{0}$.

(ii) $D^{b m}(\mathcal{W}+\widetilde{K})\left(u_{0}, y_{0}\right)(u)=D^{b m}(F+\widetilde{K})\left(u_{0}, y_{0}\right)(u)$ for any $u_{0} \in \mathbb{R}^{p}, y_{0} \in \mathcal{W}\left(u_{0}\right)$, and $u \in U$ if $F$ is $\widetilde{K}$-dominated by $\mathcal{W}$ near $u_{0}$. 


\subsection{Higher-order adjacent derivatives of perturbation maps without constraints}

Now, the relations between the higher-order adjacent derivative of feasible map and the higher-order adjacent derivative of perturbation/ weak perturbation maps are investigated in this subsection.

Proposition 4.1 Assume that $F$ is mth-order u-directionally compact at $\left(u_{0}, y_{0}\right)$ for any $u \in \mathbb{R}^{p}$.

(i) If $F$ is $K$-dominated by $\mathcal{F}$ near $u_{0}$, then, for $u$ near $u_{0}$,

$$
\operatorname{Min}_{K} D^{b m} F\left(u_{0}, y_{0}\right)(u) \subseteq D^{b m} \mathcal{F}\left(u_{0}, y_{0}\right)(u) .
$$

(ii) If $F$ is $K$-dominated by $\mathcal{P}$ near $u_{0}$, then, for $u$ near $u_{0}$,

$$
\operatorname{PrMin}_{K} D^{b m} F\left(u_{0}, y_{0}\right)(u) \subseteq D^{b m} \mathcal{P}\left(u_{0}, y_{0}\right)(u)
$$

(iii) If int $K \neq \emptyset$, there is a closed convex cone $\widetilde{K}$ satisfying $\widetilde{K} \subseteq \operatorname{int} K \cup\{0\}$, and $F$ is $\widetilde{K}$-dominated by $\mathcal{W}$ near $u_{0}$, then, for $u$ near $u_{0}$,

$$
\operatorname{WMin}_{K} D^{b m} F\left(u_{0}, y_{0}\right)(u) \subseteq D^{b m} \mathcal{W}\left(u_{0}, y_{0}\right)(u) .
$$

Proof Since the proof is similar, we prove only assertion (iii). Observe that, being a pointed closed convex cone in $\mathbb{R}^{q}, K$ clearly has a compact base and hence so does $\tilde{K}$. Moreover, $F$ is $m$ th-order $u$-directionally compact at $\left(u_{0}, y_{0}\right)$ implies that $\mathcal{W}$ is $m$ th-order $u$-directionally compact at $\left(u_{0}, y_{0}\right)$. Therefore, one has

$$
\begin{aligned}
\operatorname{WMin}_{K} D^{b m} F\left(u_{0}, y_{0}\right)(u) & =\operatorname{WMin}_{K} D^{b m}(F+\widetilde{K})\left(u_{0}, y_{0}\right)(u) \\
& =\operatorname{WMin}_{K} D^{b m}(\mathcal{W}+\widetilde{K})\left(u_{0}, y_{0}\right)(u) \\
& =\operatorname{WMin}_{K} D^{b m} \mathcal{W}\left(u_{0}, y_{0}\right)(u) \\
& \subseteq D^{b m} \mathcal{W}\left(u_{0}, y_{0}\right)(u)
\end{aligned}
$$

Here the first and third equalities are due to Propositions 3.5, and the second one follows from Remark 4.1.

Now, we investigate the reverse conclusion in Proposition 4.1.

Proposition 4.2 Suppose that the following conditions are satisfied:

(i) $F$ is locally Hölder continuous of order $m$ at $u_{0}$;

(ii) $F$ is $K$-dominated by $\mathcal{F}$ near $u_{0}$;

(iii) there is a neighborhood $U$ of $u_{0}$ such that for any $u \in U, \mathcal{F}(u)$ is a single-point set. Then, for any $u \in \mathbb{R}^{p}$,

$$
D^{b m} \mathcal{F}\left(u_{0}, y_{0}\right)(u) \subseteq \operatorname{Min}_{K} D^{b m} F\left(u_{0}, y_{0}\right)(u) .
$$

Proof Let $u \in \mathbb{R}^{p}$ and $v \in D^{b m} \mathcal{F}\left(u_{0}, y_{0}\right)(u)$. Then, for any sequence $t_{n} \downarrow 0$, there exists $\left(u_{n}, v_{n}\right) \rightarrow(u, v)$ such that

$$
y_{0}+t_{n}^{m} v_{n} \in \mathcal{F}\left(u_{0}+t_{n} u_{n}\right) \subseteq F\left(u_{0}+t_{n} u_{n}\right), \quad \forall n .
$$


Suppose to the contrary that $v \notin \operatorname{Min}_{K} D^{b m} F\left(u_{0}, y_{0}\right)(u)$. Then, there exists $\bar{v} \in D^{b m} F\left(u_{0}\right.$, $\left.y_{0}\right)(u)$ such that $v-\bar{v} \in K \backslash\{0\}$. Hence, for the preceding $t_{n}$, there exists $\left(\bar{u}_{n}, \bar{v}_{n}\right) \rightarrow(u, \bar{v})$ such that

$$
y_{0}+t_{n}^{m} \bar{v}_{n} \in F\left(u_{0}+t_{n} \bar{u}_{n}\right), \quad \forall n
$$

Since $F$ is $K$-dominated by $\mathcal{F}$ near $u_{0}$, there exists $U_{1} \in \mathcal{U}\left(u_{0}\right)$ such that, for all $u \in U_{1}$,

$$
F(u) \subseteq \mathcal{F}(u)+K .
$$

It follows from the locally Hölder continuity of order $m$ of $F$ that there exist $U_{2} \in \mathcal{U}\left(u_{0}\right)$ and $L>0$ such that, for all $u_{1}, u_{2} \in U_{2}$ and

$$
F\left(u_{1}\right) \subseteq F\left(u_{2}\right)+L\left\|u_{1}-u_{2}\right\|^{m} B_{\mathbb{R}} q
$$

Naturally, since $t_{n} \downarrow 0$, there exists $N>0$ such that

$$
u_{0}+t_{n} \bar{u}_{n}, u_{0}+t_{n} u_{n} \in U \cap U_{1} \cap U_{2}, \quad \forall n>N .
$$

Therefore, from (5), (7), (8), and (6), there exists $b_{n} \in B_{\mathbb{R}^{q}}$ such that, for all $n$ large enough,

$$
y_{0}+t_{n}^{m}\left(\bar{v}_{n}-L\left\|\bar{u}_{n}-u_{n}\right\|^{m} b_{n}\right) \in F\left(u_{0}+t_{n} u_{n}\right) \subseteq \mathcal{F}\left(u_{0}+t_{n} u_{n}\right)+K .
$$

Thus, it follows from (4), (9), and assumption (iii) that

$$
y_{0}+t_{n}^{m}\left(\bar{v}_{n}-L\left\|\bar{u}_{n}-u_{n}\right\|^{m} b_{n}\right)-\left(y_{0}+t_{n}^{m} v_{n}\right)=t_{n}^{m}\left(\bar{v}_{n}-L\left\|\bar{u}_{n}-u_{n}\right\|^{m} b_{n}-v_{n}\right) \in K .
$$

Since $\bar{v}_{n}-L\left\|\bar{u}_{n}-u_{n}\right\|^{m} b_{n}-v_{n} \rightarrow \bar{v}-v$ and $K$ is a pointed closed convex cone, one has $\bar{v}-v \in K$, which contradicts $v-\bar{v} \in K \backslash\{0\}$. This completes the proof.

The following example shows that the assumption (iii) in Proposition 4.2 cannot be dropped.

Example 4.1 Let $p=1, q=2, K=\left\{\left(y_{1}, y_{2}\right) \in \mathbb{R}^{2} \mid y_{1}=0, y_{2} \geq 0\right\},\left(u_{0}, y_{0}\right)=(0,(0,0))$, and $F: \mathbb{R} \rightrightarrows \mathbb{R}^{2}$ be defined by

$$
F(u)= \begin{cases}K, & \text { if } u=0 \\ K \cup\left\{\left(y_{1}, y_{2}\right) \in \mathbb{R}^{2} \mid y_{1}=u^{2}, y_{2} \geq-\sqrt{1+u^{2}}\right\}, & \text { if } u \neq 0\end{cases}
$$

Then

$$
\mathcal{F}(u)= \begin{cases}\{(0,0)\}, & \text { if } u=0, \\ \left\{(0,0),\left(u^{2},-\sqrt{1+u^{2}}\right)\right\}, & \text { if } u \neq 0 .\end{cases}
$$

Hence, we can check that $\mathcal{F}(u)$ is not a single-point set near $u_{0}, F$ is $K$-dominated by $\mathcal{F}$ near $u_{0}$, and $F$ is locally Hölder continuous of order 2 at $u_{0}$. By direct calculation, one has, for any $u \in \mathbb{R}$,

$$
D^{b 2} F\left(u_{0}, y_{0}\right)(u)=K \cup\left\{\left(y_{1}, y_{2}\right) \in \mathbb{R}^{2} \mid y_{1}=u^{2}\right\},
$$




$$
D^{b 2} \mathcal{F}\left(u_{0}, y_{0}\right)(u)=\{(0,0)\}
$$

and then

$$
\operatorname{Min}_{K} D^{b 2} F\left(u_{0}, y_{0}\right)(0)=\emptyset
$$

Therefore,

$$
D^{b 2} \mathcal{F}\left(u_{0}, y_{0}\right)(0) \nsubseteq \operatorname{Min}_{K} D^{b 2} F\left(u_{0}, y_{0}\right)(0) .
$$

Remark 4.2 Similar properties to Proposition 4.2 for higher-order contingent-type derivatives of perturbation maps have not yet been investigated in [31]. With some suitable modifications, we can obtain similar properties for higher-order contingent-type derivatives of perturbation maps in [31].

\section{Proposition 4.3 Suppose that the following conditions are satisfied:}

(i) $F$ is locally Hölder continuous of order $m$ at $u_{0}$;

(ii) $F$ is $K$-dominated by $\mathcal{P}$ near $u_{0}$;

(iii) there is a neighborhood $U$ of $u_{0}$ such that for any $u \in U, \mathcal{P}(u)$ is a single-point set.

Then, for any $u \in \mathbb{R}^{p}$,

$$
D^{b m} \mathcal{P}\left(u_{0}, y_{0}\right)(u) \subseteq \operatorname{PrMin}_{K} D^{b m} F\left(u_{0}, y_{0}\right)(u) .
$$

Proof The proof is similar to that of Proposition 4.2.

Proposition 4.4 Assume that int $K \neq \emptyset$. If $F$ is locally Hölder continuous of order $m$ at $u_{0}$, then, for any $u \in \mathbb{R}^{p}$,

$$
D^{b m} \mathcal{W}\left(u_{0}, y_{0}\right)(u) \subseteq \mathrm{WMin}_{K} D^{b m} F\left(u_{0}, y_{0}\right)(u)
$$

Proof Let $u \in \mathbb{R}^{p}$ and $v \in D^{b m} \mathcal{W}\left(u_{0}, y_{0}\right)(u)$. Then, for any sequence $t_{n} \downarrow 0$, there exists $\left(u_{n}, v_{n}\right) \rightarrow(u, v)$ such that

$$
y_{0}+t_{n}^{m} v_{n} \in \mathcal{W}\left(u_{0}+t_{n} u_{n}\right) \subseteq F\left(u_{0}+t_{n} u_{n}\right), \quad \forall n .
$$

Suppose to the contrary that $v \notin \mathrm{WMin}_{K} D^{b m} F\left(u_{0}, y_{0}\right)(u)$. Then there exists $\bar{v} \in D^{b m} F\left(u_{0}\right.$, $\left.y_{0}\right)(u)$ such that $v-\bar{v} \in \operatorname{int} K$. Hence, for the preceding $t_{n}$, there exists $\left(\bar{u}_{n}, \bar{v}_{n}\right) \rightarrow(u, \bar{v})$ such that

$$
y_{0}+t_{n}^{m} \bar{v}_{n} \in F\left(u_{0}+t_{n} \bar{u}_{n}\right), \quad \forall n
$$

It follows from the locally Hölder continuity of order $m$ of $F$ that there exist $U \in \mathcal{U}\left(u_{0}\right)$ and $L>0$ such that, for all $u_{1}, u_{2} \in U$, one has

$$
F\left(u_{1}\right) \subseteq F\left(u_{2}\right)+L\left\|u_{1}-u_{2}\right\|^{m} B_{\mathbb{R}} q
$$


Naturally, since $t_{n} \downarrow 0$, there exists $N>0$ such that

$$
u_{0}+t_{n} \bar{u}_{n}, u_{0}+t_{n} u_{n} \in U, \quad \forall n>N
$$

Therefore, from (12), (13), and (14), there exists $b_{n} \in B_{\mathbb{R}^{q}}$ such that, for all $n$ large enough,

$$
y_{0}+t_{n}^{m}\left(\bar{v}_{n}-L\left\|\bar{u}_{n}-u_{n}\right\|^{m} b_{n}\right) \in F\left(u_{0}+t_{n} u_{n}\right) .
$$

It follows from $v_{n}-\left(\bar{v}_{n}-L\left\|\bar{u}_{n}-u_{n}\right\|^{m} b_{n}\right) \rightarrow v-\bar{v}$ and $v-\bar{v} \in \operatorname{int} K$ that we have $v_{n}-\left(\bar{v}_{n}-\right.$ $\left.L\left\|\bar{u}_{n}-u_{n}\right\|^{m} b_{n}\right) \in \operatorname{int} K$ for $n$ large enough. Therefore, for $n$ large enough,

$$
y_{0}+t_{n}^{m} v_{n}-\left(y_{0}+t_{n}^{m}\left(\bar{v}_{n}-L\left\|\bar{u}_{n}-u_{n}\right\|^{m} b_{n}\right)\right) \in \operatorname{int} K,
$$

which contradicts with (11). The conclusion is obtained.

\subsection{Higher-order adjacent derivatives of perturbation maps with constraints}

In this section, the formulas for estimating higher-order adjacent derivative of perturbation map/ weak perturbation map via adjacent derivative of constraint map together with higher-order Fréchet derivative of the objective function are established.

Proposition 4.5 Let $u_{0} \in \mathbb{R}^{p}, x_{0} \in X\left(u_{0}\right), y_{0}=f\left(x_{0}, u_{0}\right)$. Assume that $f$ is mth-order Fréchet continuously differentiable at $\left(x_{0}, u_{0}\right)$. Then, for any $u \in \mathbb{R}^{p}$,

$$
\begin{aligned}
& \left\{y \in \mathbb{R}^{q} \mid \exists x \in D^{b} X\left(x_{0}, u_{0}\right)(u), y=d^{m} f\left(x_{0}, u_{0}\right)((x, u), \ldots,(x, u))\right\} \\
& \quad \subseteq D^{b m} F\left(u_{0}, y_{0}\right)(u) .
\end{aligned}
$$

Moreover, if $X$ is (first-order) $u$-directionally compact at $\left(u_{0}, x_{0}\right)$ for any $u \in \mathbb{R}^{p}$, then the reverse inclusion of (16) is also valid.

Proof Let $y$ be as in the left hand side of (16). Then there exist $u \in \mathbb{R}^{p}$ and $x \in$ $D^{b} X\left(x_{0}, u_{0}\right)(u)$ such that $y=d^{m} f\left(x_{0}, u_{0}\right)((x, u), \ldots,(x, u))$. Since $x \in D^{b} X\left(x_{0}, u_{0}\right)(u)$, for all $t_{n} \downarrow 0$, there exists $\left(u_{n}, x_{n}\right) \rightarrow(u, x)$ such that, for all $n, x_{0}+t_{n} x_{n} \in X\left(u_{0}+t_{n} u_{n}\right)$. Then

$$
f\left(x_{0}+t_{n} x_{n}, u_{0}+t_{n} u_{n}\right) \in F\left(u_{0}+t_{n} u_{n}\right), \quad \forall n .
$$

It follows from the $m$ th-order Fréchet continuously differentiability of $f$ and (17) that we have

$$
f\left(x_{0}, u_{0}\right)+t_{n}^{m} d^{m} f\left(x_{0}, u_{0}\right)\left(\left(x_{n}, u_{n}\right), \ldots,\left(x_{n}, u_{n}\right)\right)+o\left(t_{n}^{m}\left\|\left(x_{n}, u_{n}\right)\right\|^{m}\right) \in F\left(u_{0}+t_{n} u_{n}\right)
$$

Consequently,

$$
y_{0}+t_{n}^{m}\left(d^{m} f\left(x_{0}, u_{0}\right)\left(\left(x_{n}, u_{n}\right), \ldots,\left(x_{n}, u_{n}\right)\right)+\frac{o\left(t_{n}^{m}\left\|\left(x_{n}, u_{n}\right)\right\|^{m}\right)}{t_{n}^{m}}\right) \in F\left(u_{0}+t_{n} u_{n}\right) .
$$

It follows from (18) and

$$
d^{m} f\left(x_{0}, u_{0}\right)\left(\left(x_{n}, u_{n}\right), \ldots,\left(x_{n}, u_{n}\right)\right)+\frac{o\left(t_{n}^{m}\left\|\left(x_{n}, u_{n}\right)\right\|^{m}\right)}{t_{n}^{m}} \rightarrow d^{m} f\left(x_{0}, u_{0}\right)((x, u), \ldots,(x, u))
$$


when $n \rightarrow \infty$, one has $y=d^{m} f\left(x_{0}, u_{0}\right)((x, u), \ldots,(x, u)) \in D^{b m} F\left(u_{0}, y_{0}\right)(u)$. Hence, (16) has been established.

Now, let $y \in D^{b m} F\left(u_{0}, y_{0}\right)(u)$. Then, for all $t_{n} \downarrow 0$, there exists $\left(u_{n}, y_{n}\right) \rightarrow(u, y)$ such that $y_{0}+t_{n}^{m} y_{n} \in F\left(u_{0}+t_{n} u_{n}\right), \forall n$. Hence, there exists $x_{n} \in X\left(u_{0}+t_{n} u_{n}\right)$ such that

$$
y_{0}+t_{n}^{m} y_{n}=f\left(x_{n}, u_{0}+t_{n} u_{n}\right), \quad \forall n
$$

Setting $\widetilde{x}_{n}:=\frac{x_{n}-x_{0}}{t_{n}}$, we have

$$
x_{0}+t_{n} \widetilde{x}_{n} \in X\left(u_{0}+t_{n} u_{n}\right)
$$

and

$$
y_{0}+t_{n}^{m} y_{n}=f\left(x_{0}+t_{n} \widetilde{x}_{n}, u_{0}+t_{n} u_{n}\right), \quad \forall n
$$

Since $X$ is first-order $u$-directionally compact at $\left(u_{0}, x_{0}\right)$, for preceding $t_{n}, u_{n}$, and $\widetilde{x}_{n}$, by taking subsequence if necessary, one gets $\widetilde{x}_{n} \rightarrow \tilde{x} \in D^{b} X\left(x_{0}, u_{0}\right)(u)$. It follows from (21) and the $m$ th-order Fréchet continuously differentiability of $f$ at $\left(x_{0}, u_{0}\right)$ that one has

$$
y_{0}+t_{n}^{m} y_{n}=f\left(x_{0}, u_{0}\right)+t_{n}^{m} d^{m} f\left(x_{0}, u_{0}\right)\left(\left(\widetilde{x}_{n}, u_{n}\right), \ldots,\left(\widetilde{x}_{n}, u_{n}\right)\right)+o\left(t_{n}^{m}\left\|\left(\widetilde{x}_{n}, u_{n}\right)\right\|^{m}\right) .
$$

It implies that

$$
y_{n}=d^{m} f\left(x_{0}, u_{0}\right)\left(\left(\widetilde{x}_{n}, u_{n}\right), \ldots,\left(\widetilde{x}_{n}, u_{n}\right)\right)+\frac{o\left(t_{n}^{m}\left\|\left(\widetilde{x}_{n}, u_{n}\right)\right\|^{m}\right)}{t_{n}^{m}} .
$$

Letting $n \rightarrow \infty$, we have

$$
y=d^{m} f\left(x_{0}, u_{0}\right)((\widetilde{x}, u), \ldots,(\widetilde{x}, u)) .
$$

The proof is complete.

The following example illustrates that the assumption for the validity of the reverse inclusion of (16) in Proposition 4.5 cannot be omitted.

Example 4.2 Let $p=q=l=1, m=2, f(x, u)=x^{4}$, and $X: \mathbb{R} \rightrightarrows \mathbb{R}$ be defined by $X(u)=\{x \in$ $\mathbb{R} \mid 0 \leq x \leq 1\}$. Then $F(u)=\{y \in \mathbb{R} \mid 0 \leq y \leq 1\}$.

Let $\left(x_{0}, u_{0}\right)=(0,0)$. Then $y_{0}=f\left(x_{0}, u_{0}\right)=0$. We can check that $X$ is not $u$-directionally compact at $\left(u_{0}, x_{0}\right)$ for any $u \in \mathbb{R}$. Indeed, by taking $t_{n}=\frac{1}{n}, u_{n} \rightarrow u$, and $x_{n}=\frac{1}{2} n$, we have $x_{0}+t_{n} x_{n}=\frac{1}{2} \in F\left(u_{0}+t_{n} u_{n}\right)$, and $x_{n}$ has no convergent subsequence.

By direct calculation, one has, for any $u \in \mathbb{R}$,

$$
\begin{aligned}
& D^{b} X\left(u_{0}, x_{0}\right)(u)=\mathbb{R}_{+}, \quad D^{b 2} F\left(u_{0}, y_{0}\right)(u)=\mathbb{R}_{+} \\
& d^{2} f(x, u)=\left[\begin{array}{cc}
12 x^{2} & 0 \\
0 & 0
\end{array}\right],
\end{aligned}
$$


and then

$$
d^{2} f\left(x_{0}, u_{0}\right)=\left[\begin{array}{ll}
0 & 0 \\
0 & 0
\end{array}\right] .
$$

Hence, for any $u \in \mathbb{R}$,

$$
\left\{y \in \mathbb{R} \mid x \in D^{b} X\left(x_{0}, u_{0}\right)(u), y=d^{2} f\left(x_{0}, u_{0}\right)((x, u),(x, u))\right\}=\{0\} \varsubsetneqq D^{b 2} F\left(u_{0}, y_{0}\right)(u) .
$$

Corollary 4.1 Let $u_{0} \in \mathbb{R}^{p}, x_{0} \in X\left(u_{0}\right), y_{0}=f\left(x_{0}, u_{0}\right)$, and $\widetilde{X}: \mathbb{R}^{p} \times \mathbb{R}^{q} \rightrightarrows \mathbb{R}^{l}$ be defined by $\widetilde{X}(u, y):=\{x \in X(u): y=f(x, u)\}$. Assume that $f$ is mth-order Fréchet continuously differentiable at $\left(x_{0}, u_{0}\right)$. Let one of the following conditions be fulfilled:

(i) $X$ is locally Lipschitz calm at $\left(u_{0}, x_{0}\right) \in \operatorname{gr} X$;

(ii) $D_{S} X\left(u_{0}, x_{0}\right)(0)=\{0\}$;

(iii) $\tilde{X}$ is $(u, y)$-directionally compact at $\left(\left(u_{0}, y_{0}\right), x_{0}\right)$ for any $(u, y) \in \mathbb{R}^{p} \times \mathbb{R}^{q}$;

(iv) $\widetilde{X}$ is locally Lipschitz calm at $\left(\left(u_{0}, y_{0}\right), x_{0}\right) \in \operatorname{gr} \widetilde{X}$;

(v) $D_{S} \tilde{X}\left(\left(u_{0}, y_{0}\right), x_{0}\right)(0,0)=\{0\}$;

(vi) $\tilde{X}$ is locally pseudo-Lipschitz at $\left(\left(u_{0}, y_{0}\right), x_{0}\right)$.

Then, for any $u \in \mathbb{R}^{p}$,

$$
D^{b m} F\left(u_{0}, y_{0}\right)(u)=\left\{y \in \mathbb{R}^{q} \mid \exists x \in D^{b} X\left(x_{0}, u_{0}\right)(u), y=d^{m} f\left(x_{0}, u_{0}\right)((x, u), \ldots,(x, u))\right\} .
$$

Proof From Proposition 2.1, Corollary 2.1, Proposition 4.5 and the analysis similar the proof of Corollary 4.1 and Proposition 4.2 in [35], we obtain the conclusion.

Theorem 4.1 Let $u_{0} \in \mathbb{R}^{p}, x_{0} \in X\left(u_{0}\right), y_{0}=f\left(x_{0}, u_{0}\right)$. Suppose that the following conditions are satisfied:

(i) $F$ is mth-order u-directionally compact at $\left(u_{0}, y_{0}\right)$ for any $u \in \mathbb{R}^{p}$;

(ii) $F$ is $K$-dominated by $\mathcal{F}$ near $u_{0}$;

(iii) $F$ is locally Hölder continuous of order $m$ at $u_{0}$;

(iv) there exists $U \in \mathcal{U}\left(u_{0}\right)$ such that for any $u \in U, \mathcal{F}(u)$ is a single-point set;

(v) $f$ is mth-order Fréchet continuously differentiable at $\left(x_{0}, u_{0}\right)$;

(vi) $X$ is first-order u-directionally compact at $\left(u_{0}, x_{0}\right)$.

Then, for any $u \in \mathbb{R}^{p}$,

$$
\begin{array}{rl}
D^{b m} & \mathcal{F}\left(u_{0}, y_{0}\right)(u) \\
& =\operatorname{Min}_{K} D^{b m} F\left(u_{0}, y_{0}\right)(u) \\
& =\operatorname{Min}_{K}\left\{y \in \mathbb{R}^{q} \mid \exists x \in D^{b} X\left(x_{0}, u_{0}\right)(u), y=d^{m} f\left(x_{0}, u_{0}\right)((x, u), \ldots,(x, u))\right\} .
\end{array}
$$

Proof It follows from Proposition 4.1(i), Proposition 4.2, and Proposition 4.5 that the proof is complete.

The result in Theorem 4.1 is illustrated in the following example.

Example 4.3 Let $p=q=l=1, m=2, K=\mathbb{R}_{+}, f(x, u)=x^{2}$, and $X: \mathbb{R} \rightrightarrows \mathbb{R}$ be defined by $X(u)=\left\{x \in \mathbb{R} \mid u^{2} \leq x \leq 2 u^{2}\right\}$. Then

$$
F(u)=\left\{y \in \mathbb{R} \mid u^{4} \leq y \leq 4 u^{4}\right\},
$$




$$
\mathcal{F}(u)=\left\{u^{4}\right\}
$$

Let $\left(x_{0}, u_{0}\right)=(0,0)$. Then $y_{0}=f\left(x_{0}, u_{0}\right)=0$. It is easy to see that the assumptions (ii) and (iv) in Theorem 4.1 are satisfied. Since $D_{S}^{2} F\left(u_{0}, y_{0}\right)(0)=\{0\}$, from the Proposition 2.1, the assumption (i) Theorem 4.1 is fulfilled.

Moreover, we can check that $F$ is locally Hölder continuous of order 2 at $u_{0}$ and $X$ is first-order $u$-directionally compact at $\left(u_{0}, x_{0}\right)$. Hence, the assumptions (iii) and (vi) in Theorem 4.1 are fulfilled.

By direct calculation, one has, for any $u \in \mathbb{R}$,

$$
\begin{aligned}
& D^{b} X\left(u_{0}, x_{0}\right)(u)=\{0\}, \quad D^{b 2} F\left(u_{0}, y_{0}\right)(u)=\{0\}, \\
& D^{b 2} \mathcal{F}\left(u_{0}, y_{0}\right)(u)=\{0\}, \\
& d^{2} f(x, u)=\left[\begin{array}{ll}
2 & 0 \\
0 & 0
\end{array}\right],
\end{aligned}
$$

and then

$$
d^{2} f\left(x_{0}, u_{0}\right)((x, u),(x, u))=2 x^{2}
$$

Thus, all the assumptions in Theorem 4.1 are satisfied. For any $u \in \mathbb{R}$ and $x \in D^{b} X\left(u_{0}\right.$, $\left.x_{0}\right)(u)$, i.e., $x=0$, one has

$$
d^{2} f\left(x_{0}, u_{0}\right)((x, u),(x, u))=0
$$

Hence, for any $u \in \mathbb{R}$,

$$
\begin{array}{rl}
D^{b 2} & \mathcal{F}\left(u_{0}, y_{0}\right)(u) \\
& =\operatorname{Min}_{K} D^{b 2} F\left(u_{0}, y_{0}\right)(u) \\
& =\operatorname{Min}_{K}\left\{y \in \mathbb{R} \mid x \in D^{b} X\left(x_{0}, u_{0}\right)(u), y=d^{2} f\left(x_{0}, u_{0}\right)((x, u),(x, u))\right\} .
\end{array}
$$

Theorem 4.2 Let $u_{0} \in \mathbb{R}^{p}, x_{0} \in X\left(u_{0}\right), y_{0}=f\left(x_{0}, u_{0}\right)$. Suppose that the following conditions are satisfied:

(i) $F$ is mth-order u-directionally compact at $\left(u_{0}, y_{0}\right)$ for any $u \in \mathbb{R}^{p}$;

(ii) $F$ is $K$-dominated by $\mathcal{P}$ near $u_{0}$;

(iii) $F$ is locally Hölder continuous of order $m$ at $u_{0}$;

(iv) there exists $U \in \mathcal{U}\left(u_{0}\right)$ such that for any $u \in U, \mathcal{P}(u)$ is a single-point set;

(v) $f$ is mth-order Fréchet continuously differentiable at $\left(x_{0}, u_{0}\right)$;

(vi) $X$ is first-order $u$-directionally compact at $\left(u_{0}, x_{0}\right)$.

Then, for any $u \in \mathbb{R}^{p}$,

$$
\begin{array}{rl}
D^{b m} & \mathcal{P}\left(u_{0}, y_{0}\right)(u) \\
& =\operatorname{PrMin}_{K} D^{b m} F\left(u_{0}, y_{0}\right)(u) \\
& =\operatorname{PrMin}_{K}\left\{y \in \mathbb{R}^{q} \mid \exists x \in D^{b} X\left(x_{0}, u_{0}\right)(u), y=d^{m} f\left(x_{0}, u_{0}\right)((x, u), \ldots,(x, u))\right\} .
\end{array}
$$


Proof It follows from Proposition 4.1(ii), Proposition 4.3, and Proposition 4.5 that the conclusion is obtained.

Theorem 4.3 Let $u_{0} \in \mathbb{R}^{p}, x_{0} \in X\left(u_{0}\right), y_{0}=f\left(x_{0}, u_{0}\right)$. Suppose that the following conditions are satisfied:

(i) $F$ is mth-order $u$-directionally compact at $\left(u_{0}, y_{0}\right)$ for any $u \in \mathbb{R}^{p}$;

(ii) $\operatorname{int} K \neq \emptyset$, there is a closed convex cone $\widetilde{K}$ satisfying $\widetilde{K} \subseteq \operatorname{int} K \cup\{0\}$ and $F$ is $\widetilde{K}$-dominated by $\mathcal{W}$ near $u_{0}$;

(iii) $F$ is locally Hölder continuous of order $m$ at $u_{0}$;

(iv) $f$ is mth-order Fréchet continuously differentiable at $\left(x_{0}, u_{0}\right)$;

(v) $X$ is $u$-directionally compact at $\left(u_{0}, x_{0}\right)$.

Then, for any $u \in \mathbb{R}^{p}$,

$$
\begin{array}{rl}
D^{b m} & \mathcal{W}\left(u_{0}, y_{0}\right)(u) \\
& =\operatorname{WMin}_{K} D^{b m} F\left(u_{0}, y_{0}\right)(u) \\
& =\operatorname{WMin}_{K}\left\{y \in \mathbb{R}^{q} \mid \exists x \in D^{b} X\left(x_{0}, u_{0}\right)(u), y=d^{m} f\left(x_{0}, u_{0}\right)((x, u), \ldots,(x, u))\right\} .
\end{array}
$$

Proof It follows from Proposition 4.1(iii), Proposition 4.4, and Proposition 4.5 that the conclusion is given.

\section{Conclusions}

Although there are some similar properties between the contingent derivatives and the adjacent derivatives, the adjacent derivatives have some advantages and drawbacks in some cases. When using adjacent derivatives instead of contingent derivatives in sensitivity analysis, the proto-differentiability assumption, such as in Theorem 3.3 in [4], Theorem 4.3 in [17], Theorem 5.1 in [18], can be omitted. The drawbacks of using of the adjacent derivatives is that the adjacent derivatives can be empty set in some cases such as in Example 2.1. Hence, in the case that the adjacent derivatives are not empty and avoiding the proto-differentiability assumption, the adjacent derivatives can be used. Based on the above observation, the $m$ th-order adjacent derivatives was employed to consider higherorder sensitivity analysis for nonsmooth vector optimization in this paper. First of all, we considered the relationships between the $m$ th-order adjacent derivatives of the perturbation map/the proper perturbation map/the weak perturbation map, and the $m$ th-order adjacent derivative of feasible map in objective space. Then the above relations were used to establish the formulas for estimating the $m$ th-order adjacent derivatives of the perturbation map, the proper perturbation map, and the weak perturbation map via the adjacent derivative of constraint map and the $m$ th-order Fréchet derivative of the objective map. Some examples are provided to ensure the need of the assumptions and illustrate the results. When $m=1$, the results become the first-order sensitivity analysis using adjacent derivatives and also may be new. 
Received: 31 December 2015 Accepted: 1 April 2016 Published online: 08 April 2016

\section{References}

1. Tanino, T: Sensitivity analysis in multiobjective optimization. J. Optim. Theory Appl. 56, 479-499 (1988)

2. Tanino, T: Stability and sensitivity analysis in convex vector optimization. SIAM J. Control Optim. 26, $521-536$ (1988)

3. Shi, DS: Contingent derivative of the perturbation map in multiobjective optimization. J. Optim. Theory Appl. 70, 385-396 (1991)

4. Kuk, H, Tanino, T, Tanaka, M: Sensitivity analysis in vector optimization. J. Optim. Theory Appl. 89, 713-730 (1996)

5. Balbás, A, Jiménez Guerra, P: Sensitivity analysis for convex multiobjective programming in abstract spaces. J. Math. Anal. Appl. 202, 645-648 (1996)

6. Jiménez Guerra, P, Melguizo, MA, Muñoz, MJ: Sensitivity analysis in convex programming. Comput. Math. Appl. 58, 1239-1246 (2009)

7. Kuk, H, Tanino, T, Tanaka, M: Sensitivity analysis in parameterized convex vector optimization. J. Math. Anal. Appl. 202, 511-522 (1996)

8. Shi, DS: Sensitivity analysis in convex vector optimization. J. Optim. Theory Appl. 77, 145-159 (1993)

9. Chuong, TD, Yao, J-C: Generalized Clarke epiderivatives of parametric vector optimization problems. J. Optim. Theory Appl. 146, 77-94 (2010)

10. Garcia, F, Melguizo Padial, MA: Sensitivity analysis in convex optimization through the circatangent derivative. J. Optim. Theory Appl. 165, 420-438 (2015)

11. Jiménez Guerra, P, Melguizo Padial, MA: Sensitivity analysis in differential programming through the Clarke derivative. Mediterr. J. Math. 9, 537-550 (2012)

12. Rockafellar, RT: Proto-differentiablility of set-valued mappings and its applications in optimization. Ann. Inst. Henri Poincaré, Anal. Non Linéaire 6, 449-482 (1989)

13. Levy, AB, Rockafellar, RT: Sensitivity analysis of solutions to generalized equations. Trans. Am. Math. Soc. 345, 661-671 (1994)

14. Levy, AB, Rockafellar, RT: Variational conditions and the proto-differentiation of partial subgradient mappings. Nonlinear Anal. 26, 1951-1964 (1996)

15. Lee, GM, Huy, NQ: On proto-differentiablility of generalized perturbation maps. J. Math. Anal. Appl. 324, 1297-1309 (2006)

16. Lee, GM, Huy, NQ: On sensitivity analysis in vector optimization. Taiwan. J. Math. 11, 945-958 (2007)

17. Wang, QL, Li, SJ: Second-order contingent derivative of the pertubation map in multiobjective optimization. Fixed Point Theory Appl. 2011, Article ID 857520 (2011)

18. Wang, QL, Li, SJ: Sensitivity and stability for the second-order contingent derivative of the proper perturbation map in vector optimization. Optim. Lett. 6, 731-748 (2012)

19. Li, SJ, Liao, CM: Second-order differentiability of generalized perturbation maps. J. Glob. Optim. 52, 243-252 (2012)

20. Tung, LT: Second-order radial-asymptotic derivatives and applications in set-valued vector optimization. Pac. J. Optim. (accepted for publication)

21. Tung, LT: On second-order proto-differentiability of perturbation maps (submitted for publication)

22. Sun, XK, Li, SJ: Stability analysis for higher-order adjacent derivative in parametrized vector optimization. J. Inequal. Appl. 2010, Article ID 510838 (2010)

23. Tung, LT: Higher-order contingent derivatives of perturbation maps in multiobjective optimization. J. Nonlinear Funct. Anal. 2015, Article ID 19 (2015)

24. Khanh, PQ, Tuan, ND: Variational sets of multivalued mappings and a unified study of optimality conditions. J. Optim. Theory Appl. 139, 45-67 (2008)

25. Anh, NLH, Khanh, PQ: Variational sets of perturbation maps and applications to sensitivity analysis for constrained vector optimization. J. Optim. Theory Appl. 158, 363-384 (2013)

26. Frankowska, H: An open mapping principle for set-valued maps. J. Math. Anal. Appl. 127, 172-180 (1987)

27. Frankowska, H, Quincampoix, M: Hölder metric regularity of set-valued maps. Math. Program. 132, 333-345 (2012)

28. Studniarski, M: Necessary and sufficient conditions for isolated local minima of nonsmooth functions. SIAM J. Control Optim. 25, 1044-1049 (1986)

29. Studniarski, M, Ward, D: Weak sharp minima: characterizations and sufficient condition. SIAM J. Control Optim. 38 219-236 (1999)

30. Anh, NLH, Khanh, PQ, Tung, LT: Higher-order radial derivatives and optimality conditions in nonsmooth vector optimization. Nonlinear Anal. TMA 74, 7365-7379 (2011)

31. Diem, HTH, Khanh, PQ, Tung, LT: On higher-order sensitivity analysis in nonsmooth vector optimization. J. Optim. Theory Appl. 162, 463-488 (2014)

32. Sun, XK, Li, SJ: Weak lower Studniarski derivative in set-valued optimization. Pac. J. Optim. 8, 307-320 (2012)

33. Sun, XK, Li, SJ: Lower Studniarski derivative of the perturbation map in parameterized vector optimization. Optim. Lett. 5, 601-614 (2011)

34. Anh, NLH, Khanh, PQ: Calculus and applications of Studniarski's derivatives to sensitivity and implicit function theorems. Control Cybern. 43, 35-47 (2014)

35. Aubin, JP, Frankowska, H: Set-Valued Analysis. Birkhäuser, Boston (1990) 